\title{
Molecular characterization and gene silencing of Laccase 1 in the grain aphid, Sitobion avenae
}

\author{
Yong Zhang ${ }^{1,2}$ iD | Jia Fan ${ }^{1}$ | Frédéric Francis ${ }^{2}$ | Julian Chen ${ }^{1}$
}

\author{
${ }^{1}$ State Key Laboratory of Plant Diseases and \\ Insect Pests, Institute of Plant Protection, \\ Chinese Academy of Agricultural Sciences, Bei- \\ jing, P.R. China \\ ${ }^{2}$ Functional and Evolutionary Entomology, Gem- \\ bloux Agro-Bio Tech, University of Liège, Liege, \\ Belgium \\ Correspondence \\ Julian Chen, State Key Laboratory of Plant Dis- \\ eases and Insect Pests, Institute of Plant Protec- \\ tion, Chinese Academy of Agricultural Sciences, \\ Beijing 100193, P.R. China. \\ Email:jlchen@ippcaas.cn
}

\begin{abstract}
Laccase 1 (Lac1), a polyphenol oxidase, has been proposed to be involved in insect iron metabolism and immunity responses. However, little information is available on the roles of Lac 1 in insectplant interactions. The grain aphid Sitobion avenae is one of the most destructive pests of cereal, directly drawing phloem sap and transmitting viruses. In the present study, we first cloned the open reading frame (ORF) of Lac 1 from S. avenae, and the putative protein sequence was predicted to have a carboxyl-terminal transmembrane domain. We found that SaLac1 had higher expression levels in the fourth and adult stages using reverse transcription real-time quantitative PCR (RT-qPCR). SaLac 1 was highly expressed in the salivary gland and midgut and also in wingless compared with winged morphs. After feeding on aphid-resistant wheat with a high total phenol content, the expression level of SaLac 1 increased significantly. RNA interference (RNAi) by oral feeding successfully inhibited the transcript levels of SaLac 1, and the knockdown of Lac 1 significantly decreased the survival rate of $S$. avenae on aphid-resistant wheat. Our study demonstrated that S. avenae Lac1 was involved in the detoxification of phenolic compounds in wheat and was essential for the aphid to adapt to resistant plants.
\end{abstract}

\section{KEYWORDS}

aphid-wheat interactions, laccase 1, RNAi, RT-qPCR, Sitobion avenae

\section{1 | INTRODUCTION}

Laccases, members of the multicopper oxidase (MCO) family, are polyphenol oxidases found in plants, fungi, bacteria, and insects (Alexandre \& Zhulin, 2000; Bao, O'Malley, Whetten, \& Sederoff, 1993; Pezet, Pont, \& Hoangvan, 1991). Laccases can oxidize a wide range of compounds, including diphenols, monolignols, isoflavonoids, and tannins (Mayer \& Staples, 2002). Two types of laccase genes, laccase 1 (Lac 1) and laccase 2 (Lac 2), are identified in many insect species (Dittmer et al., 2004; Parkinson et al., 2003). Several studies demonstrate that Lac 2 encodes an enzyme that is principally involved in insect cuticular pigmentation and hardening (Futahashi, 2011; Niu et al., 2008 ). Lac 2 is highly expressed in insect cuticles and levels of expression correlate temporally and spatially with cuticle sclerotization. 
Knockdown of Lac 2 using an RNA interference (RNAi) technique impaired insect cuticle tanning (Eliasneto, Soares, Simões, Hartfelder, \& Bitondi, 2010; Futahashi, 2011). However, silencing of the Lac 1 gene in the red flour beetle, Tribolium castaneum, by injection of dsRNA had no effects on cuticle tanning (Arakane, Muthukrishnan, Beeman, Kanost, \& Kramer, 2005).

Orthologous Lac 1 genes have been identified in many insect species, including the whitefly, Bemisia tabaci (Yang et al., 2017); green rice leafhopper, Nephotettix cincticeps (Hattori et al., 2010), and pea aphid Acyrthosiphon pisum (Liang, 2006). Lac 1 is involved in iron homeostasis and immune defense of insects. Expression levels of orthologous Lac 1 in the fruit fly, Drosophila melanogaster, increased with septic injury, indicating the role of Lac 1 in the melanization pathway during the immunity response of the insect (Gregorio, Spellman, Rubin, \& Lemaitre, 2001). The transcript levels of MCO 1 are upregulated in midgut and Malpighian tubules of the mosquito Anopheles gambiae in response to a blood meal, which is either a source of iron or an injection of bacteria, suggesting that MCO 1 is involved in either iron metabolism or immunity (Gorman, Dittmer, Marshall, \& Kanost, 2008; Liu et al., 2015). Additionally, Lac1 has been detected in some insect tissues that function as detoxification systems, such as midguts and salivary glands; therefore, Lac 1 is also hypothesized to be involved in the oxidation of toxic phenolic compounds ingested by insects during feeding and play an important role in insect-plant interactions (Dittmer et al., 2004). However, little research has been conducted to verify this hypothesis.

The grain aphid, Sitobion avenae, is the major pest of cereal in world, causing a severe yield loss from 20 to $80 \%$ by both direct feeding phloem sap and transmitting plant viruses, such as barley yellow dwarf virus (BYDV) (Blackman \& Eastop, 2000; Fereres, Gutierrez, Del Estal, \& Castañera, 1988). The enzyme activity of polyphenol oxidases has been detected in the saliva of S. avenae (Ma, Chen, Cheng, \& Sun, 2010). The primary goal of this study was to determine the potential roles of Lac1 in the interactions between S. avenae and wheat. In this study, we first obtained the complete open reading frame (ORF) sequence and then determined the temporal and spatial expression patterns of Lac 1 in $S$. avenae using RT-qPCR. Next, to determine whether Lac1 was involved in the interaction with plants or the detoxification of toxic secondary plant metabolites, the transcript levels of Lac1 in the aphid were detected after feeding on aphid-resistant wheat with high total phenol content. Knockdown of Lac1 in S. avenae by feeding on dsRNA and the subsequent effects on aphid survival were also determined to further examine the role of this gene in aphid-wheat interactions.

\section{2 | MATERIALS AND METHODS}

\subsection{Plants and aphids}

Seeds of aphid-susceptible winter wheat, Triticum aestivum var. Beijing 837 (BJ837) and aphid-resistant wheat var. KOK1679 (Chen, Sun, Ding, Ni, \& Li, 1997), were respectively immersed in 0.5\% sodium hypochlorite (Amresco, OH, USA) for $30 \mathrm{~min}$ to sterilize the surface, then washed three times, and germinated in distilled water for 3-4 days at a temperature of $25 \pm 1^{\circ} \mathrm{C}$ in sterilized petri dishes. Seedlings with similar size were carefully transferred into plastic plots with organic soil, and rearing continued in a climate chamber (16-h light:8-h dark; $20 \pm 1^{\circ} \mathrm{C}$ ).

Clones of $S$. avenae were initially established from a single aphid collected from a wheat field in Langfang City, Hebei Province, northern China and have been reared on aphid-susceptible wheat plants (var. BJ 837) for at least 6 years (25-30 generations per year) in an indoor environment at a temperature of $20 \pm 1^{\circ} \mathrm{C}$, relative humidity of $75 \%$ and photoperiod of 16-h light:8-h dark.

\subsection{Sequence and phylogenetic analysis}

The full ORF of Lac 1 was obtained from the transcriptome of $S$. avenae (unpublished). The protein sequence was deduced using ORF finder (https://www.ncbi.nlm.nih.gov/orffinder/). The molecular weight and isoelectric point of the deduced protein sequence were calculated using ExPASy Proteomics Server 
TAB LE 1 Primers used for RT-qPCR analysis of SaLac 1 and reference genes

\begin{tabular}{|lllll|}
\hline Genes & Primer sequences & PCR product size $(\mathbf{b p})$ & Amplification efficiency (E)\% & $R^{2}$ values \\
\hline SaLac 1 & Forward: GACCAGACTGTGTGCCGGC & 159 & 99 & 0.9996 \\
& Reverse: TTACCGTGCCAGTGGACAGA & & \\
\hline -Actin & Forward: CGTTACCAACTGGGACGATATG & 111 & 96 & 0.9994 \\
& Reverse: GGGTTCAATGGAGCTTCTGTTA & & 0.9918 \\
\hline NADH & Forward: CGAGGAGAACATGCTCTTAGAC & 93 & 113 & \\
\hline & Reverse: GATAGCTTGGGCTGGACATATAG & &
\end{tabular}

(http://cn.expasy.org/tools/pi_tool.html). Signal peptide cleavage sites and transmembrane helices region in the protein sequence were predicted with SignalP 4.1 Server (http://www.cbs.dtu.dk/services/SignalP/) and TMHMM Server v 2.0 (http://www.cbs.dtu.dk/services/TMHMM/), respectively. The presence or absence of glycosylphosphatidylinositol (GPI) anchor sites was predicted with GPI-SOM (http://gpi.unibe.ch/). Cu-oxidase Pfam domains in the sequence were predicted using SMART (http://smart.embl-heidelberg.de/).

The phylogenetic tree was constructed with 46 laccase sequences from insects, plants, fungi, and bacteria using the neighbor-joining method with a matrix of pair-wise distances estimated by a Poisson model for amino acid sequences through MEGA 5.05 software. Gaps were treated by the pairwise deletion method; bootstrap values were calculated on 1,000 replications; branch points with bootstrap values less than $50 \%$ were collapsed.

\section{3 | Expression profiles of SaLac 1 in different aphid tissues, developmental stages, and morphs}

Approximately 600 salivary glands and 300 midguts of wingless adult aphids were dissected in phosphate buffered saline $(\mathrm{pH}=7.2)$. All samples were transferred into liquid nitrogen immediately and stored at $-70^{\circ} \mathrm{C}$ until used. Total RNA was extracted from different tissues, instars, and morphs of aphids using TRIzol Reagent (Invitrogen, CA, USA) following the protocols provided by the manufacturer. The quality and quantity of RNA were assessed with NanoDrop 2000 Spectrophotometer (Thermo Scientific, CA, USA). A total of $1 \mu \mathrm{g}$ of RNA was reverse transcribed into cDNA with a transcript one-step gDNA removal and cDNA synthesis supermix kit (TransGenBiotech, Beijing, China) following the manufacturer's instructions, and CDNA templates were stored at $-20^{\circ} \mathrm{C}$.

RT-qPCR was conducted on an ABI 7500 Real-Time PCR System (Applied Biosystems, CA, USA). The cDNA templates were diluted to 10-fold and then used as templates to detect the relative expression of the target genes in a $20 \mu \mathrm{L}$ reaction system containing $2 \mu \mathrm{L}$ of cDNA, $0.5 \mu \mathrm{L}$ of $10 \mu \mathrm{mol} / \mathrm{L}$ forward primer and reverse primer each, $10 \mu \mathrm{L}$ of $2 \times$ SYBR premix Ex Taq ${ }^{\mathrm{TM}}$ (Tli RNaseH Plus, Takara, Dalian, China), and $0.4 \mu \mathrm{L}$ of $50 \times$ ROX Reference Dye II at the following conditions: $30 \mathrm{~s}$ at $95^{\circ} \mathrm{C}$ followed by 40 cycles of $30 \mathrm{~s}$ at $95^{\circ} \mathrm{C}$ and $40 \mathrm{~s}$ at $60^{\circ} \mathrm{C}$. In the RT-qPCR, three biological replicates were analyzed for each sample, each replicate consisted of three technical replicates and the differential expression was calculated using the $2^{-\Delta \Delta C t}$ method (Livak \& Schmittgen, 2012). The primers of target gene SaLac 1 and two reference genes $\beta$-actin and NADH dehydrogenase (NADH) (Xue et al., 2016) are presented in Table 1.

\subsection{Transcript levels of SaLac 1 in aphids after feeding on aphid-susceptible and resistant wheat plants}

Wingless adults of $S$. avenae were first transferred onto new wheat plants; the next day, only newborn nymphs were left on the plants, which were reared to wingless adults. At the two-leaf stage, five synchronous wingless adults of $S$. avenae were transferred to the first leaf of BJ 837 (aphid-susceptible) or KOK1679 (aphid-resistant) and restricted in a plastic ecological cage $(2.7 \mathrm{~cm} \times 2.7 \mathrm{~cm} \times 2.7 \mathrm{~cm})$ to prevent aphid escape. The edge of ecological cages was covered with sponge to avoid causing mechanical wounding of the leaf. Each pot contained one wheat plant and was grown in climate incubator at $20 \pm 1^{\circ} \mathrm{C}$ and a photoperiod of 16-h light:8-h dark. After 24 or $48 \mathrm{~h}$ of feeding, all aphids were 
TAB LE 2 Primers used for dsRNA synthesis of SaLac 1 and GFP

\begin{tabular}{cll} 
Genes & Primer sequences & PCR product size (bp) \\
\hline dsLac 1 & Forward: TAATACGACTCACTATAGGGCAGAATTAGAAGACGCAACA & 613 \\
\hline & Reverse: TAATACGACTCACTATAGGG CCTCAACGTGGAACTCAA & \\
\hline dsGFP & Forward: TAATACGACTCACTATAGGG TACGGCGTGCAGTGCT & 495 \\
\hline & Reverse: TAATACGACTCACTATAGGG TGATCGCGCTTCTCG \\
\hline
\end{tabular}

collected, and RT-qPCR was performed as described before to detect the expression of SaLac 1 after feeding on wheat. Each treatment was a set of three replicates, and aphids that fed on BJ 837 were used as control group.

\subsection{RNA interference of SaLac 1 by feeding on dsRNA}

PCR primers with T7 promoter sequences were used to produce the SaLac 1 gene for further dsRNA synthesis (Table 2). dsRNA was generated and purified using a MEGAscript T7 Transcription Kit (Thermo Fisher Scientific, CA, USA) following the manufacturer's protocols. The dsRNA was detected by agarose gel electrophoresis and quantified using a NanoDrop 2000 Spectrophotometer (Thermo Fisher Scientific). The dsRNA was stored at $-80^{\circ} \mathrm{C}$ until further use. Sucrose solution, 30\%, was formulated as aphid artificial diet, and the feeding apparatus was prepared according to a previous report (Chen, Ni, Ding, \& Sun, 2000). Briefly, dsRNA was first diluted into artificial diet at 50, 100, and $200 \mathrm{ng} / \mu \mathrm{L}$. Two hundred microliters of artificial diet with dsLac 1 or dsGFP (control) was sandwiched between two layers of parafilm membrane. Wingless adult aphids were collected from fresh wheat plants, and then 30 healthy and active aphids were assigned into treatment and control groups with three replicates in each group, after being starved for $3 \mathrm{~h}$. Each feeding device was placed in an artificial climate chamber with a temperature of $20 \pm 1^{\circ} \mathrm{C}$, humidity of $75 \%$, and photoperiod of 16-h light:8-h dark. All survived aphids were collected at days 1 and 3 of feeding on dsRNA at different dsRNA concentrations to detect the inhibition efficiency of Lac1 transcription in S. avenae.

\subsection{Detection of aphid survival after SaLac 1 gene silencing}

After feeding on $200 \mathrm{ng} / \mu \mathrm{L}$ dsRNA for 3 days, 20 wingless adult aphids were fed wholly on freshly prepared pure artificial diet (without any dsRNA) or transferred onto aphid resistant wheat plants (var. KOK1679). The artificial diet was replaced every third day to prevent mildew. The number of surviving aphids was recorded for seven consecutive days, and the effect of SaLac 1 gene silencing on aphid survival was calculated as the percentage of surviving aphids to the total aphids. Each treatment consisted of three replicates.

\subsection{Statistical analyses}

The relative expression of SaLac 1 in different aphid tissues and developmental stages was calculated with that of whole wingless adults as reference. The expression of SaLac 1 in wingless adults was calculated using that of winged adults as reference. The effect of dsRNA at different concentrations on SaLac 1 expression was expressed relative to that of control groups. All results of RT-qPCR were analyzed using the SPSS Statistic 17.0 software package (SPSS, Inc., CHI, USA), and the differences between or among groups were examined using $t$ tests or one-way analysis of variance. The survival rate of aphids on artificial diet or wheat was analyzed using the log-rank (Mantel-Cox) test. $P$ values less than 0.05 indicated statistical significance.

\section{3 | RESULTS}

\subsection{CDNA cloning and sequence analysis of SaLac 1}

As shown in Figure 1, the ORF of S. avenae Lac 1 (SaLac 1) contained 2,157 bases encoding 718 amino acid residues with a predicted molecular weight of $10.82 \mathrm{kDa}$. The GenBank accession number of SaLAC1 is MG189702. The N-terminal 
ATGAGATCACAGTGCGCCACCACCGTCGTGCTGTTCTACTACTGCTGCTGTACGATCGCCGTGCTGCGCTTGACGGGCGCCGTGAAGCCGCGACACGAACGCGGACAGGAGGACTACAAC

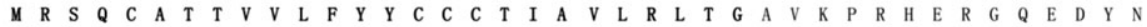

121 GCCGTCCATCCTTGCCAGAGAGAATGTCGCGCGGGCGAACCGCCCAAAACGTGCGAGTACCGTTTCAAAGTGGAATGGTACTACACAATGAGCAAGGCGTGCTACGACTGTCCGTACAAC

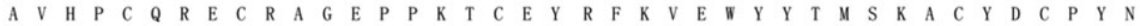

241 ATGACCGATTGTTACAGACCAGACTGTGTGCCGGCCGACGGCGTTGCGAAGCCCATTATCGTCATCAATAGAAGTCTGCCGGGACCTTCCATACAAGTGTGCTTGGGCGACACGGTCATG M T D

361 GTGGACGTGGAGAACGCCATGATGGAGGAGTCGACGTCTGTCCACTGGCACGGTCACCACCAGCGCAACTCACCGTACATGGACGGCGTGCCGTACGTGACACAGTGTCCGGTTCCGCCG

GTGGACGTGGAGAACGCCATGATGGAGGAGTCGACGTCTGTCCACTGGCACGGTCACCACCAGCGCAACTCACCGTACATGGACGGCGTGCCGTACGTGACACAGTGTCCGGTTCCGCC

481 CACAGTTCGTTCCGGTACGCGTACCTGGCCGACAACGAGGGCACGCACTTCTGGCACTCGCATTCCGGCTGCCAGCGGGGTGACGGAGCGTTCGGGTCGTTCGTGGTACGCGCCCCAAAG

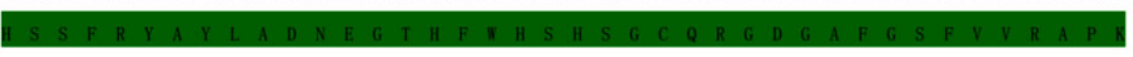

601 TCGCGGGACGTGCACCGCGACATGTACGACGTGGACGTGCACGTCATCACGGTCACCGACTGGCTGCACGAGCTGGGCATCCGCAAGTTCCTCGCCCATTACCACGGTTCGGGCAACAAC I $\begin{array}{lllllllllllllllllll} & R & D & V & H & R & D & M & Y & D & V\end{array}$

721 AagCCCGAGACCATTCTGATAAACGGCCGCGGCCGTTACAAAGTCTTCGACGGCGGTTACCGCACGCCCCTCACTCAGTTCAACGTGACCAGAGGGAAACGATATAGGTTTAGACTGATA

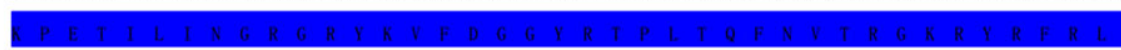

841 AaCGCTGGATTCCTAAATTGTCCAATTTCAATGAGTATCGACAATCATACGTTTACAATGATTGCAACAGATGGATATAACGTTCAACCAGTAGTAGTCGATTCGTTTGTAAGTTATGCT

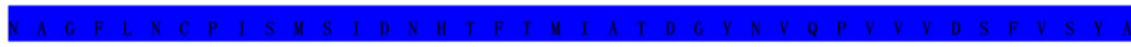

961 GGTGAACGCTGGGATTTTGTTGTGGAAGCGACTGCTAATGTTGGCAATTATTGGATGCGATTTAGAGGCTTGATGGATTGCGATGAACGGTTCACTAAGGCCTTTGAAGTATCGATTTTG

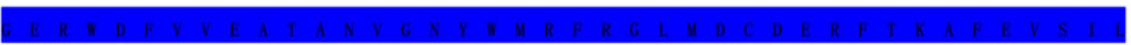

1081 CATTATGACGgAGCTGGTGACGAGGAACCAGAGgGCACACCAACATATGACAATACATTTCATTCTGGAATTCAATTGAATGCACTGAATAAAGGATCTGGGTTAATGGACACTGCTACT

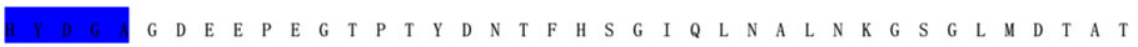

1201 GTATCAGAATTAGAAGACGCAACACCCCCAAAAAATGACCTTCGTTTGGAGAAAAAACCAGATGTAACATTATTTATGTCATATGATTTTTATTCTTTAGATAATCCACATTTCCACAAA

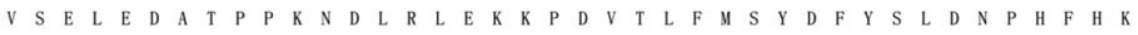

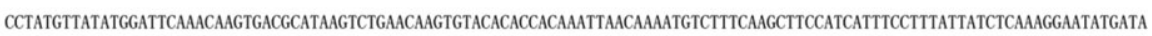

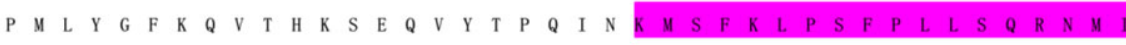

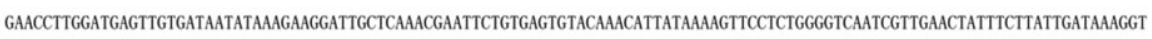

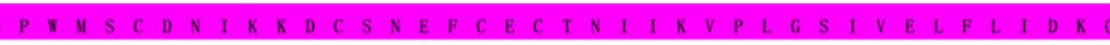

GTGACCTATAACGCAAATCATCCATTCCATTTGCACGGACATCCGTTTAGAGTAGTAGCGATGGAAAGAGTTGGAAATCATACTACTGTAGAGgAGATAGAACAAATGGACAGAGATGGG $\begin{array}{lllllllllllllllllllllllllllllllllllllll}T & Y & N & A & N & H & P & F & H & L & H & G & H & P & F & R & V & V & A & \text { H } & E & R & V & G & N & H & T & T & V & E & E & I & E & Q & H & D & R & D & G\end{array}$

CGCATTGTGAGAAATCTACGGACTGCACCTCTTAAAGACACCGTTACAGTACCAGATGGCGGATTTACAATATTACGATTCTTGGCCGACAATCCTGGTTATTGGTTATTTCATTGTCAT

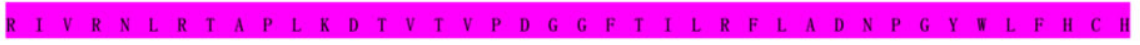

1801 ATTGAGTTCCACGTTGAGGTGGGCATGGCAACTGTGTTTAAAATCGGCGAAGACTGGGAAATGCCCCCATCACCTCCGGGTTTTCCGAAATGTGGAAACTATAACGGAAAAGGATTAGTA

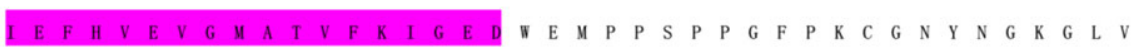

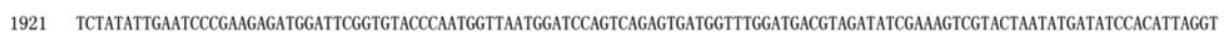

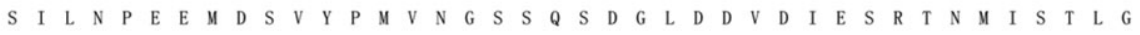

2041 AAATGGTGGCCATTCGTCGGAGGTGCGCATCCTTATGTAGCATCTGCTGCTAGTATTAACTCATCGTATTTTTCAATTTTATTGTGTATTGTGATAAGTTTTTTAGTTATTGAATGA

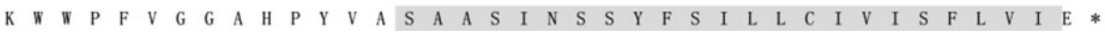

FIGURE 1 Full-length ORF sequence and deduced amino-acid sequence of SaLac1 (GenBank accession no. MG189702)

Notes. The putative signal peptide predicted by SignalP 4.1 is bold and underlined. Sequence region with green, blue, and pink color represent T2, T1, and T3 copper domains, respectively. Carboxyl-terminal transmembrane region is indicated with gray color.

signal peptide of 27 amino acid residues was predicted using SignalP 4.1. The protein sequence was predicted to have a carboxyl-terminal transmembrane region and be GPI-anchored; therefore, the expectation was that SaLac 1 was attached to the exterior of the plasma membrane through a carboxyl-terminal transmembrane region or a GPI anchor. The encoded protein had three typical Cu-oxidase domains, located in 84-201, 212-365, and 463-618 amino acid residues. 


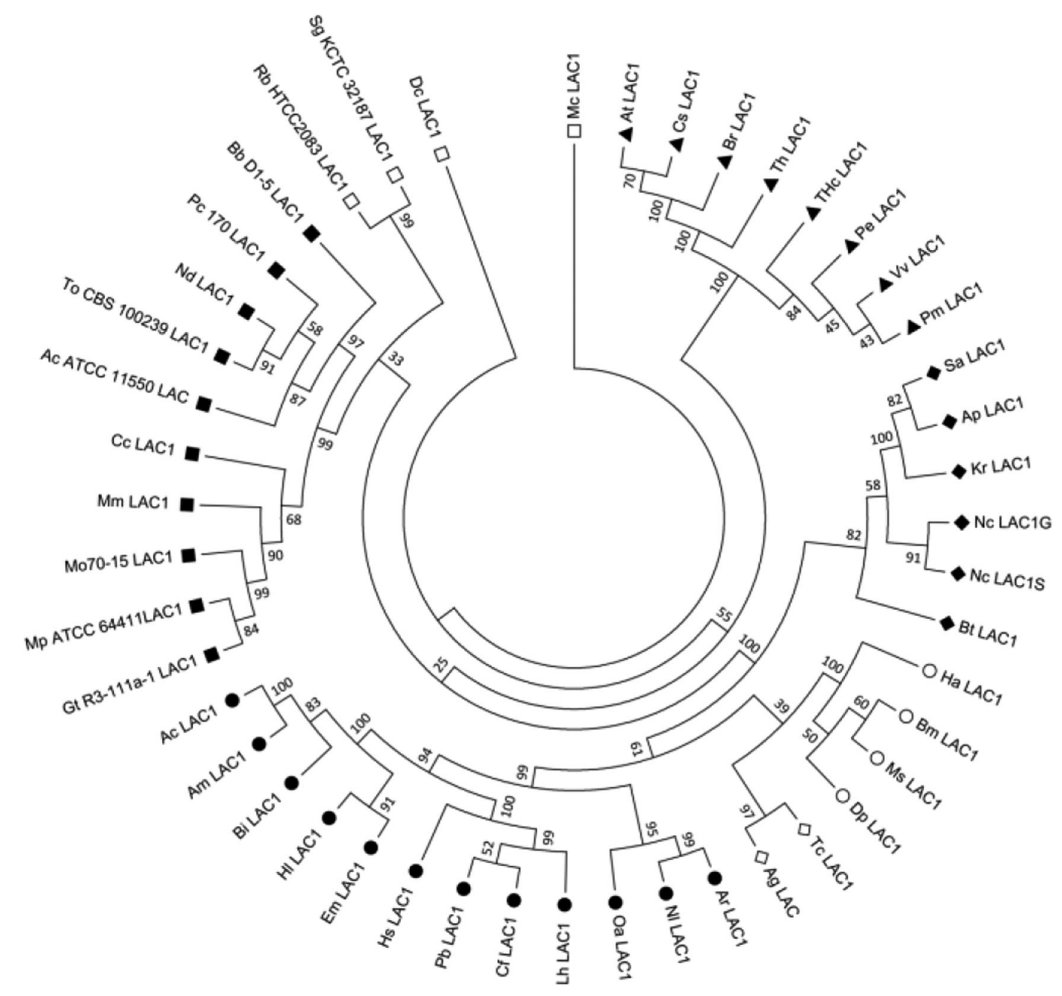

FIGURE 2 Phylogenetic tree constructed by comparing the amino-acid sequences of SaLac 1 and known Lac 1 from plants, fungi, bacteria, and insects

Notes. Phylogenetic tree was constructed by the neighbor-joining method using MEGA5.05. Bootstrap values calculated as a percentage for 1,000 replications are shown at nodes. All Lac 1 amino acid sequences and abbreviations used to generate the tree are listed in Table S1. Solid triangle, diamond, circle, and square indicated plants, hemipetrans, hymenopterans, and fungi. Hollow circle, diamond, and square represented lepidopterans, coleopterans, and bacteria.

\subsection{Phylogenetic analysis}

The phylogenetic tree was constructed to study the evolutionary relationships among Lac 1 genes of plants, insects, fungi, and bacteria. As shown in Figure 2, Lac 1 genes were clustered into independent clades according to taxonomic classification. Lac 1 gene of S. avenae had a close evolutionary relation with that of A. pisum, B. tabaci, N. cincticeps, and Du-ensiform gall aphid Kaburagia rhusicola, which are all hemipteran insects.

\subsection{Expression profiles of SaLac1 in different developmental stages, tissues, and morphs}

The temporal and spatial expression of SaLac 1 was detected using RT-qPCR (Figure 3A-C). Based on the results, SaLac1 was detected in all developmental stages of $S$. avenae. The expression levels of SaLac 1 were lowest at the pseudo-embryo stage and then were upregulated to the highest levels at the fourth ( $1.98 \pm 0.15$-fold) and adult stages $(1.87 \pm 0.05$-fold; $P<0.001)$. SaLac 1 had a higher level of expression in wingless adults than that in winged adults $(1.98$ \pm 0.16 -fold; $P=0.027$ ), and the transcript levels of SaLac 1 were most highly expressed in the salivary gland (2.88 \pm 0.28 -fold) and midgut of aphids (1.62 \pm 0.13 -fold; $P<0.001$ ).

\subsection{Expression of SaLac 1 after feeding on aphid-susceptible and resistant wheat}

No significant differences were detected in the expression levels of SaLac 1 after feeding on aphid-susceptible (BJ 837) and resistant (KOK1679) wheat for $24 \mathrm{~h}$ (Figure 4). Additionally, no significant differences were observed in gene 
A

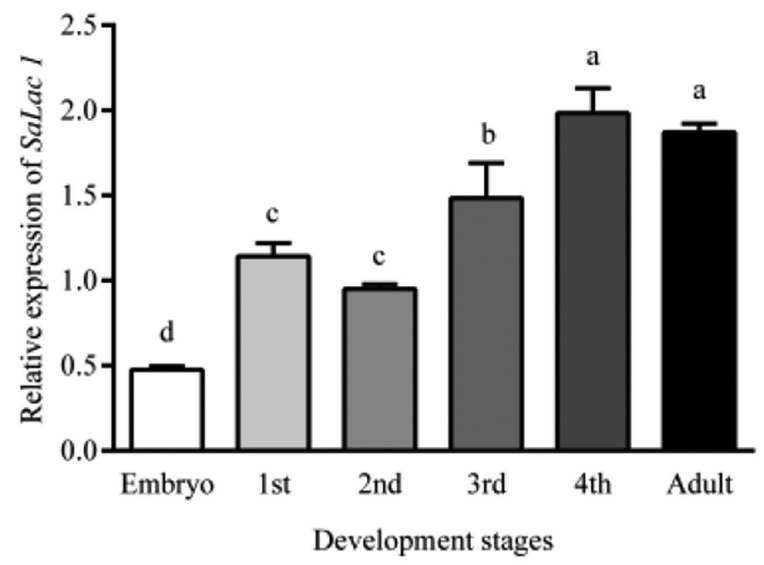

$\mathrm{B}$

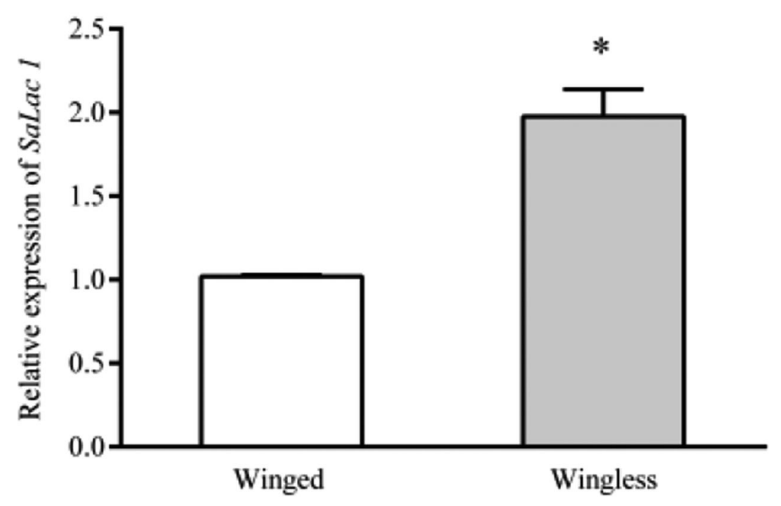

$\mathrm{C}$

Morphs

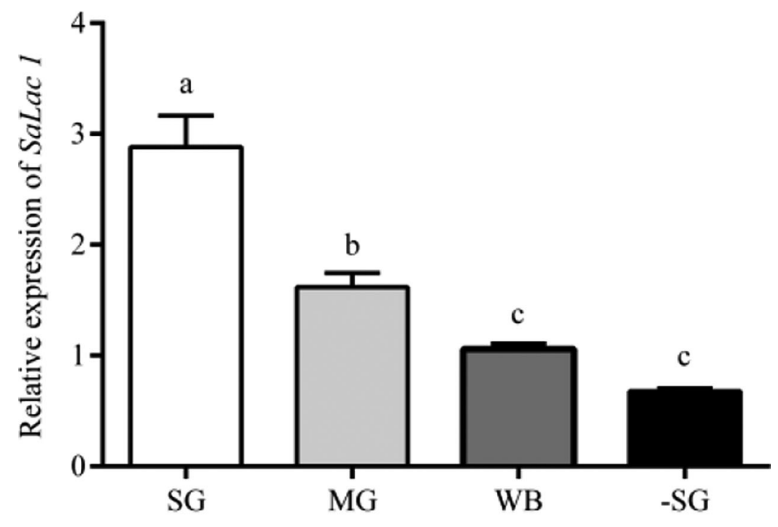

Tissues

FIGURE 3 Temporal and spatial expression of SaLac 1 analyzed by RT-qPCR

Notes. (A) Relative expression of SaLac 1 in pseudo-embryo, larval, and adult stages. (B) Relative expression of SaLac 1 in winged and wingless morphs. (C) Relative expression of SaLac 1 in salivary gland (SG), midgut (MG), whole body (WB), and whole body removed salivary gland (-SG). The bars indicate Mean \pm SE. The different lowercase letters and asterisk indicate significant difference $(P<0.05)$. 


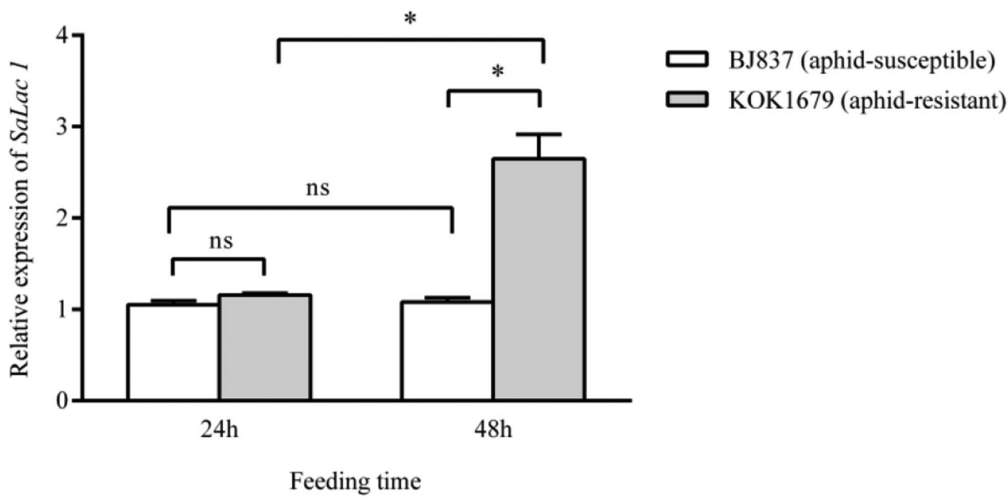

FIGURE 4 Relative expression of Lac 1 in Sitobion avenae after feeding on aphid-susceptible and aphid-resistant wheat

Notes. The bars indicate Mean \pm SE. Asterisk indicates significant difference between groups $(P<0.05)$. No statistically difference between groups is indicated as "ns."

TA B LE 3 Relative expression of SaLac 1 in Sitobion avenae after feeding on dsRNA for 1 and 3 days

\begin{tabular}{|c|c|c|c|c|}
\hline Feeding time & dsRNA & $50 \mathrm{ng} \mu \mathrm{L}^{-1}$ & $100 \mathrm{ng} \mu \mathrm{L}^{-1}$ & $200 \mathrm{ng} \mu \mathrm{L}^{-1}$ \\
\hline \multirow[t]{2}{*}{1 day } & dsGFP & $0.94 \pm 0.056$ & $1.06 \pm 0.040$ & $1.07 \pm 0.076$ \\
\hline & dsLac 1 & $0.95 \pm 0.053$ & $0.95 \pm 0.063$ & $0.70 \pm 0.026^{*}$ \\
\hline \multirow[t]{2}{*}{3 days } & dsGFP & $1.11 \pm 0.070$ & $1.09 \pm 0.054$ & $1.05 \pm 0.056$ \\
\hline & dsLac 1 & $1.17 \pm 0.06$ & $0.64 \pm 0.040^{*}$ & $0.49 \pm 0.039 *$ \\
\hline
\end{tabular}

The values (mean $\pm \mathrm{SE}$ ) in the table indicate silencing efficiencies for the different target gene dsRNA treatments relative to the control group (dsGFP-treated group). Asterisk indicates significant difference between groups $(P<0.05)$.

expression when exposed to BJ837 for 24 and 48 h. However, the expression levels of SaLac 1 were significantly upregulated ( $2.65 \pm 0.27$-fold; $P=0.025)$ after feeding on aphid-resistant wheat for $48 \mathrm{~h}$.

\subsection{Expression levels of SaLac 1 after feeding on dsRNA}

The silencing efficiency of different concentrations of dsRNA on the SaLac 1 gene was examined using RT-qPCR. As shown in Table 3, the transcript levels of SaLac 1 in S. avenae decreased significantly after feeding on $200 \mathrm{ng} / \mu \mathrm{L} \mathrm{dsLac}$ 1 for 1 day $(P=0.011)$. After 3 days treatment, the expression of SaLac 1 was significantly inhibited when exposed to $100 \mathrm{ng} / \mu \mathrm{L}(P=0.003)$ and $200 \mathrm{ng} / \mu \mathrm{L} d s \mathrm{Lac} 1(P=0.001)$.

\subsection{Effect of SaLac 1 silencing on aphid survival}

The effect of knockdown of SaLac 1 on aphid survival was determined. First, aphids were fed $200 \mathrm{ng} / \mu \mathrm{L} d s L a c 1$ for 3 days to knockdown the target gene and then were transferred onto either artificial diet or aphid-resistant wheat. As shown in Figure 5A, the survival rate of S. avenae treated with dsLac1 was not significantly different from the control group (dsGFP) when fed the artificial diet. However, the survival rate of S. avenae treated with dsLac 1 decreased to 58.3 $\pm 1.67 \%$ after feeding on aphid-resistant wheat for 4 days, significantly lower than that of the control group $(P=0.003)$ and was further reduced to $40.0 \pm 2.88 \%$ at day $7(P=0.001$; Figure $5 B)$.

\section{4 | DISCUSSION}

First, Laccase 1 was obtained from S. avenae in our study, and three different conserved copper domains, Type-1 (T1), Type-2 (T2), and Type-3 (T3) (Dwivedi, Singh, Pandey, \& Kumar, 2011), were found in the sequence, indicating that SaLac 


\section{A}

Artificial diet

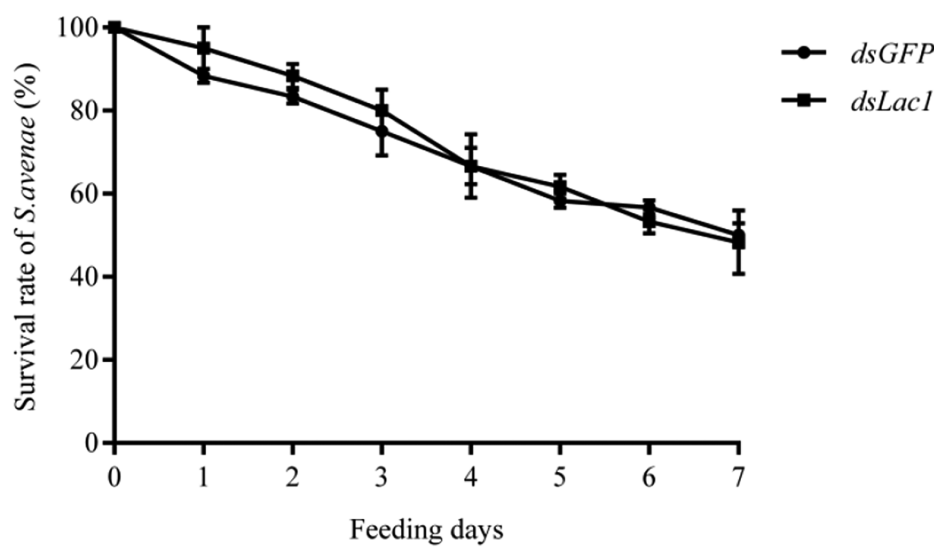

B

KOK1679

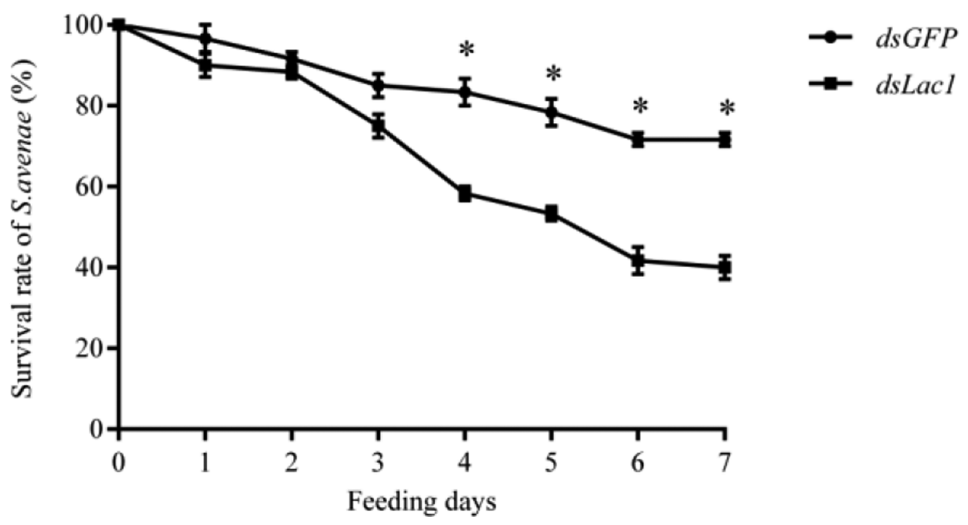

FIGURE 5 Effects of SaLac 1 silencing on the survival rate of Sitobion avenae

Notes. (A) Survival rate of S. avenae fed on artificial diet after exposed to dietary dsLac 1 and dsGFP. (B) Survival rate of $S$. avenae fed on aphid-resistant wheat after exposed to dietary dsLac 1 and dsGFP. Asterisk indicates significant difference between groups $(P<0.05)$.

1 is a member of the blue copper-containing polyphenol oxidase family and has oxidative activity. Studies demonstrate that Lac 1 is a secreted protein, and laccase activity has been detected in the watery saliva of $B$. tabaci and $N$. cincticeps (Hattori, Konishi, Tamura, Konno, \& Sogawa, 2005; Yang et al., 2017). However, in our study, Lac 1 in S. avenae was predicted as attached to the exterior of the plasma membrane. Phylogenetic analysis showed that Lac 1 cloned from S. avenae was clustered into the same branch as other hemipterans. Thus, the Lac 1 gene is evolutionarily related within insects and may have similar physiological functions.

SaLac 1 was detected in all developmental stages of S. avenae, suggesting that this enzyme plays critical roles in aphid development. The expression of SaLac 1 reached the highest levels at the fourth and adult stages, which was possibly related to increased food consumption. Aphids might improve the detoxifying abilities for secondary metabolites in phloem sap by upregulating the expression levels of SaLac 1. Yang et al. (2017) also found that Lac 1 had the highest levels of expression in the fourth instar and adult stage of $B$. tabaci.

Lac 1 transcripts have been detected in the salivary gland and midgut of A. pisum and B. tabaci (Liang, 2006; Yang et al., 2017). NcLac1S and NcLac1G, two isoforms of Lac 1, were identified in N. cincticeps; NcLac1S was expressed exclusively in the salivary glands, whereas NcLac1G was detected in the cuticle, Malpighian tubules, and midgut, in addition 
to in the salivary glands (Hattori et al., 2010). Our results showed that SaLac 1 was most highly expressed in the salivary gland and midgut of $S$. avenae. The insect salivary gland and midgut are two important sites for detoxification of toxic compounds; thus, the tissue expression patterns were consistent with predicted functions of Lac 1 as a detoxifying enzyme.

To determine whether SaLac 1 was involved in interactions with wheat and the detoxification of toxic phenolic compounds, the expression level of SaLac 1 in S. avenae was detected after feeding on aphid-resistant wheat variety KOK1679. The contents of total phenol are positively correlated with wheat resistance to aphids, and research shows that KOK1679 has high total phenol content as an antibiosis resistant cultivar (Chen et al., 1997). The expression of SaLac 1 was significantly induced after feeding on KOK1679, indicating that Lac 1 of S. avenae was involved in the detoxification of phenolic compounds in wheat.

To further demonstrate the potentially important roles of Lac 1 in interactions with plants, we performed feedingbased RNAi to knockdown the expression of SaLac 1 and then detected the effects of gene silencing on aphid survival. The data presented in this study showed that oral delivery of gene-specific dsRNA successfully silenced the target gene in S. avenae. In previous studies, catalase, odor-binding proteins and genes in guts of $S$. avenae were successfully silenced by feeding on dsRNA (or siRNA) (Deng \& Zhao, 2014; Fan et al., 2015; Zhang et al., 2013), indicating that RNAi by oral feeding is a reliable and efficient method for the functional analysis of genes in $S$. avenae and further aphid control.

RNAi induced by oral delivery of dsRNA is often less efficient than that induced through microinjection (Rajagopal, Sivakumar, Agrawal, Malhotra, \& Bhatnagar, 2002). Some studies suggest that dsRNA is degraded in artificial diets with salivary secretions and hemolymph, resulting in a low efficiency of gene silencing (Christiaens, Swevers, \& Smagghe, 2014). In our study, knockdown of the target gene failed at the low concentration of dsRNA at day 1 , but the expression of SaLac 1 was inhibited successfully when aphids were fed with the high concentration of dsRNA for longer treatment duration. The efficiency of oral feeding-based RNAi is associated with feeding time and dsRNA concentration (Baum et al., 2007; Chen et al., 2010). Therefore, increased feeding times and high dsRNA concentrations may remedy the effects of dsRNA degradation. However, studies also show that high concentrations fail to increase silencing (Atsushi et al., 2007; Shakesby et al., 2009); therefore, the optimal concentration of dsRNA for high silencing efficiency must be determined.

We found that the survival rate of S. avenae with Lac 1 silencing was not affected after feeding on pure artificial diet, but decreased significantly after feeding on aphid-resistant wheat KOK1679 at day 4 . These results suggested that Lac 1 of $S$. avenae was involved in interactions with wheat and was essential for successful adaptation to wheat resistance.

In addition to the detoxification of phenolic compounds, Lac 1 may be involved in the formulation of the stylet sheath (Hattori et al., 2005). During the process of probing and feeding, aphids secrete saliva into plant cells, and a mixture of saliva and phloem sap flows simultaneously into salivary glands. The salivary Lac 1 of S. avenae is hypothesized to use phenolic compounds in wheat plants as substrate and promote the rapid oxidative gelling of the stylet sheath via the quinine tanning reaction, which is a hypothesis worthy of further study.

In conclusion, Lac 1 was first cloned from S. avenae. High levels of expression were found in the fourth and adult stages, and SaLac 1 was also highly expressed in the salivary gland and midgut of the aphid. The results of RT-qPCR and RNAi indicated that SaLac 1 had a potentially important role in overcoming plant resistance by detoxifying toxic phenolic metabolites.

\section{ACKNOWLEDGMENTS}

We would like to thank the technician, Yanxia Liu for aphid rearing. This work was funded by National Natural Science Foundation of China (nos. 31371946, 31401740), National Key R \& D Plan in China (nos. 2016YFD0300701, 2017YFD-0201701), and Cooperation Project between China and Belgium from Ministry of Science and Technology (MOST) (no. 2014DFG32270). The funders had no roles in the study design, data collection and analysis, decision to publish, or preparation of the manuscript. 


\section{ORCID}

Yong Zhang (1D) http://orcid.org/0000-0003-4134-2405

\section{LITERATURE CITED}

Alexandre, G., \& Zhulin, I. B. (2000). Laccases are widespread in bacteria. Trends in Biotechnology, 18, 41-42.

Arakane, Y., Muthukrishnan, S., Beeman, R.W., Kanost, M. R., \& Kramer, K. J. (2005). Laccase 2 is the phenoloxidase gene required for beetle cuticle tanning. Proceedings of the National Academy of Sciences of United States of America, 102, 1133711342.

Atsushi, M., Noriaki, S., Kazuhiro, K., Masahiro, S., Kennichi, S., \& Tooru, S. (2007). RNA interference suggests sulfakinins as satiety effectors in the cricket Gryllus bimaculatus. Journal of Insect Physiology, 53, 840-848.

Bao, W., O'Malley, D. M., Whetten, R., \& Sederoff, R. R. (1993). A laccase associated with lignification in loblolly pine xylem. Science, 260, 672-674.

Baum, J.A., Bogaert, T., Clinton, W., Heck, G. R., Feldmann, P., Ilagan, O., ... Pleau, M. (2007). Control of coleopteran insect pests through RNA interference. Nature Biotechnology, 25, 1322-1326.

Blackman, R. L., \& Eastop, V. F. (2000). Aphids on the world's crops: An identification and information guide. New York: John Wiley $\&$ Sons Ltd.

Chen, J., Zhang, D., Yao, Q., Zhang, J., Dong, X., Tian, H., ... Zhang, W. (2010). Feeding-based RNA interference of a trehalose phosphate synthase gene in the brown planthopper, Nilaparvata lugens. Insect Molecular Biology, 19, 777-786.

Chen, J. L., Ni, H. X., Ding, H. J., \& Sun, J. R. (2000). Studies on a chemically defined diet of English grain aphid. Scientia Agricultura Sinica, 33, 54-59.

Chen, J. L., Sun, J. R., Ding, H. J., Ni, H. X., \& Li, X. F. (1997). The resistant patterns and mechanism of biochemical resistance in various wheat cultivars (lines). Acta Entomologica Sinica, 40, 190-195.

Christiaens, O., Swevers, L., \& Smagghe, G. (2014). DsRNA degradation in the pea aphid (Acyrthosiphon pisum) associated with lack of response in RNAi feeding and injection assay. Peptides, 53, 307-314.

Deng, F., \& Zhao, Z. W. (2014). Influence of catalase gene silencing on the survivability of Sitobion avenae. Archives of Insect Biochemistry and Physiology, 86, 46-57.

Dittmer, N. T., Suderman, R. J., Jiang, H., Zhu, Y. C., Gorman, M. J., Kramer, K. J., \& Kanost, M. R. (2004). Characterization of cDNAs encoding putative laccase-like multicopper oxidases and developmental expression in the tobacco hornworm, Manduca sexta, and the malaria mosquito, Anopheles gambiae. Insect Biochemistry and Molecular Biology, 34, $29-41$.

Dwivedi, U. N., Singh, P., Pandey, V.P., \& Kumar, A. (2011). Structure-function relationship among bacterial, fungal and plant laccases. Journal of Molecular Catalysis B Enzymatic, 68, 117-128.

Eliasneto, M., Soares, M. P., Simões, Z. L., Hartfelder, K., \& Bitondi, M. M. (2010). Developmental characterization, function and regulation of a Laccase 2 encoding gene in the honey bee, Apis mellifera (Hymenoptera, Apinae). Insect Biochemistry and Molecular Biology, 40, 241-251.

Fan, J., Zhang, Y., Francis, F., Cheng, D. F., Sun, J. R., \& Chen, J. L. (2015). Orco mediates olfactory behaviors and winged morph differentiation induced by alarm pheromone in the grain aphid, Sitobion avenae. Insect Biochemistry and Molecular Biology, 64, 16-24.

Fereres, A., Gutierrez, C., Del Estal, P., \& Castañera, P. (1988). Impact of the English grain aphid, Sitobion avenae (F.) (Homoptera: Aphididae), on the yield of wheat plants subjected to water deficits. Environmental Entomology, 17, 596-602.

Futahashi, R. (2011). Laccase2 is required for cuticular pigmentation in stinkbugs. Insect Biochemistry and Molecular Biology, 41 , 191-196.

Gorman, M. J., Dittmer, N. T., Marshall, J. L., \& Kanost, M. R. (2008). Characterization of the multicopper oxidase gene family in Anopheles gambiae. Insect Biochemistry and Molecular Biology, 38, 817-824.

Gregorio, E. D., Spellman, P. T., Rubin, G. M., \& Lemaitre, B. (2001). Genome-wide analysis of the Drosophila immune response by using oligonucleotide microarrays. Proceedings of the National Academy of Sciences of United States of America, 98, 1259012595.

Hattori, M., Konishi, H., Tamura, Y., Konno, K., \& Sogawa, K. (2005). Laccase-type phenoloxidase in salivary glands and watery saliva of the green rice leafhopper, Nephotettix cincticeps. Journal of Insect Physiology, 51, 1359-1365.

Hattori, M., Tsuchihara, K., Noda, H., Konishi, H., Tamura, Y., Shinoda, T., ... Hasegawa, T. (2010). Molecular characterization and expression of laccase genes in the salivary glands of the green rice leafhopper, Nephotettix cincticeps (Hemiptera: Cicadellidae). Insect Biochemistry and Molecular Biology, 40, 331-338. 
Liang, Q. (2006). Laccase-1 in the pea aphid, Acyrthosiphon pisum (Harris). Manhattan, NY: Kansas State University.

Liu, X. M., Sun, C. X., Liu, X., Yin, X. G., Wang, B. H., Du, M. F., \& An, S. H. (2015). Multicopper oxidase-1 is required for iron homeostasis in Malpighian tubules of Helicoverpa armigera. Scientific Reports, 5, 345-346.

Livak, K. J, \& Schmittgen, T.D. (2012). Analysis of relative gene expression data using real-time quantitative PCR and the $2^{-\Delta \Delta C T}$ Method. Methods, 25, 402-408.

Ma, R., Chen, J. L, Cheng, D. F, \& Sun, J. R. (2010). Activation of defense mechanism in wheat by polyphenol oxidase from aphid saliva. Journal of Agricultural and Food Chemistry, 58, 2410-2418.

Mayer, A. M, \& Staples, R. C. (2002). Laccase: New functions for an old enzyme. Cheminform, 60, 551-565.

Niu, B. L., Shen, W. F., Liu, Y., Weng, H. B., He, L. H., Mu, J. J., ... Meng, Z. Q. (2008). Cloning and RNAi-mediated functional characterization of MaLac2 of the pine sawyer, Monochamus alternatus. Insect Molecular Biology, 17, 303-312.

Parkinson, N. M., Conyers, C. M., Keen, J. N., Macnicoll, A. D., Smith, I., \& Weaver, R. J. (2003). cDNAs encoding large venom proteins from the parasitoid wasp Pimpla hypochondriaca identified by random sequence analysis. Comparative Biochemistry and Physiology Part C: Toxicology and Pharmacology, 134, 513-520.

Pezet, R., Pont, V., \& Hoangvan, K. (1991). Evidence of oxidative detoxication of pterostilbene and resveratrol by a laccase-like stilbene oxidase produced by Botrytis cinerea. Physiological and Molecular Plant Pathology, 39, 441-450.

Rajagopal, R., Sivakumar, S., Agrawal, N., Malhotra, P., \& Bhatnagar, R. K. (2002). Silencing of midgut aminopeptidase N of Spodoptera litura by double-stranded RNA establishes its role as Bacillus thuringiensis toxin receptor. Journal of Biological Chemistry, 277, 46849-46851.

Shakesby, A. J., Wallace, I. S., Isaacs, H. V., Pritchard, J., Roberts, D. M., \& Douglas, A. E. (2009). A water-specific aquaporin involved in aphid osmoregulation. Insect Biochemistry and Molecular Biology, 39, 1-10.

Xue, W. X., Fan, J., Zhang, Y., Xu, Q. X., Han, Z. L., Sun, J. R., \& Chen, J. L. (2016). Identification and expression analysis of candidate odorant-binding protein and chemosensory protein genes by antennal transcriptome of Sitobion avenae. PLoS One, 11 , e161839.

Yang, C. H., Guo, J. Y., Chu, D., Ding, T. B., Wei, K. K., Cheng, D. F., \& Wan, F. H. (2017). Secretory laccase 1 in Bemisia tabaci MED is involved in whitefly-plant interaction. Scientific Reports, 7, 3623.

Zhang, M., Zhou, Y. W., Wang, H., Jones, H., Gao, Q., Wang, D. H., ... Xia, L. Q. (2013). Identifying potential RNAi targets in grain aphid (Sitobion avenae F.) based on transcriptome profiling of its alimentary canal after feeding on wheat plants. BMC Genomics, 14, 560. https://doi.org/10.1186/1471-2164-14-560

\section{SUPPORTING INFORMATION}

Additional Supporting Information may be found online in the supporting information tab for this article.

How to cite this article: Zhang Y, Fan J, Francis F, Chen J. Molecular characterization and gene silencing of Laccase 1 in the grain aphid, Sitobion avenae. Arch Insect Biochem Physiol. 2018;e21446. https://doi.org/10.1002/arch.21446 